\title{
Meniscus dynamics in bubble formation: A parametric study
}

\author{
Petr Stanovsky ${ }^{\mathrm{a}, *}$, Marek C. Ruzicka ${ }^{\mathrm{a}}$, Artur Martins ${ }^{\mathrm{b}}$, Jose A. Teixeira ${ }^{\mathrm{b}}$ \\ a Institute of Chemical Process Fundamentals, Department of Multiphase Reactors, Academy of Sciences of the Czech Republic, Rozvojova 135, 16502 Prague, Czech Republic \\ ${ }^{\mathrm{b}}$ Center of Biological Engineering, IBQF, University of Minho, 4710-057 Braga, Portugal
}

\section{A R T I C L E I N F O}

\section{Article history:}

Received 15 October 2010

Received in revised form

1 February 2011

Accepted 2 February 2011

Available online 7 February 2011

\section{Keywords:}

Bubble formation

Transparent perforated plate

Meniscus oscillations

Dynamic coupling

\begin{abstract}
A B S T R A C T
This study brings new experimental data on the bubble formation process with a particular focus on the behaviour of the gas-liquid interface (meniscus) inside a transparent orifice on a perforated plate. The meniscus plays an important role by coupling the gas and liquid sides. The goal was to find the effect of five important control parameters on the bubble production and the meniscus behaviour (orifice diameter, plate thickness, gas chamber size, liquid height, and liquid viscosity). The gas input was also varied. Three physical signals were taken in the measurements to extract the information needed (gas pressure in plenum, acoustic pressure in liquid, and video record of meniscus motion). Several relevant quantities were measured to characterize the bubbling process (bubbling period, active orifice time, and periods of meniscus oscillations). The data obtained show how these quantities depend on the control parameters. The recognition of the role of the meniscus dynamics in the gas dispersing process with perforated plates offers a deeper insight into this important and complicated problem.
\end{abstract}

c) 2011 Elsevier Ltd. All rights reserved.

\section{Introduction}

The bubble formation at submerged orifices is one of the essential processes taking place in gas-liquid reactors as bubble columns, airlifts, aerated bioreactors, and wastewater activation tanks. The gas distributor (e.g. perforated plate sparger) has a crucial importance for the flow character in the apparatus, on the prevailing flow regime (e.g. homogeneous/heterogeneous), and on the regime transition. It determines the bubble size distribution, the local intensity of sparging, whence the features of the bubbly driven flow. Experiments with variety of gas spargers have been performed in the past (e.g. Deckwer, 1992; Kastanek et al., 1993). However, there is still no decisive guide for the gas distributor design. Interplay between the way of sparging and the resulting bubbly flow structure need further attention (Mudde, 2005). There is a considerable amount of work about bubble formation and its modelling, covered by several reviews (e.g. Valentin, 1967; Kumar and Kuloor, 1970; Clift et al., 1978; Tsuge, 1986; Kulkarni and Joshi, 2005).

Nevertheless, still there are a lot of questions about the detailed bubble formation dynamics. Recently, we focused on the behaviour of the gas-liquid meniscus inside the orifice whose oscillations between the subsequent bubbles detachment had a strong impact on the bubbling modes. Both experiments (Ruzicka et al., 2009a) and mathematical modelling (Ruzicka et al., 2009b;

\footnotetext{
* Corresponding author. Tel.: +420 220390 384; fax: +420 220920661 .

E-mail address: stanovsky@icpf.cas.cz (P. Stanovsky).
}

Ruzicka, 2009) were done, to capture the main essence of the nonlinear meniscus dynamics, under fixed operation parameters. The objectives of present work is to continue in this direction by performing a parametric study to see the trends of the system response to the variation of few important parameters related to geometry (orifice diameter, plate thickness, gas chamber volume, and liquid height) and material properties (liquid viscosity). The references to other literature sources were made previously and are not repeated here.

\section{Experiments}

The experimental apparatus was a building-block system of completely transparent parts, to see the motion of the meniscus inside the orifice, see Fig. 1a. The water container was a rectangular $9 \mathrm{~cm} \times 13 \mathrm{~cm}$ glass box of two heights, 13 and $80 \mathrm{~cm}$. The gas plenum was a $7 \mathrm{~cm}$ diameter Plexiglas cylinder of four heights, $1,3,5$, and $8 \mathrm{~cm}$. The plenum bottom was equipped with a porous plate to ensure the uniform distribution of the inflowing gas. The perforated plate with a single circular orifice had the thickness $L$ varying from 3 to $12 \mathrm{~mm}$. The orifice diameter $d$ was from 0.5 to $5 \mathrm{~mm}$. The gas was the filtered air (condensation dryer Donaldson, activated carbon Ultrafilter AG-AK, and microfiber filters AG-SMF). The liquids were distilled water (conductivity $\sim 1 \mathrm{mS} / \mathrm{cm}$ ) and aqueous solutions of glycerol (p.a. LachNer). The temperature was kept nearly constant, at about $23{ }^{\circ} \mathrm{C}$ in all experiments. 
The experimental setup is drawn in Fig. 1b. The gas flows at the controlled rate $Q$ (Bronkhorst, type F-201-FA-22-V) through the gas plenum (volume $V$ ) and through the orifice in the plate (dimensions $L, d$ ) into the liquid (height $H$, density $\rho$, viscosity $\mu$ ),

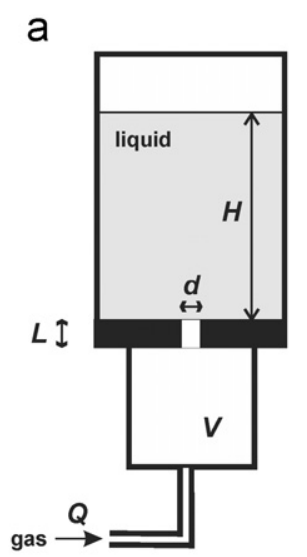

b

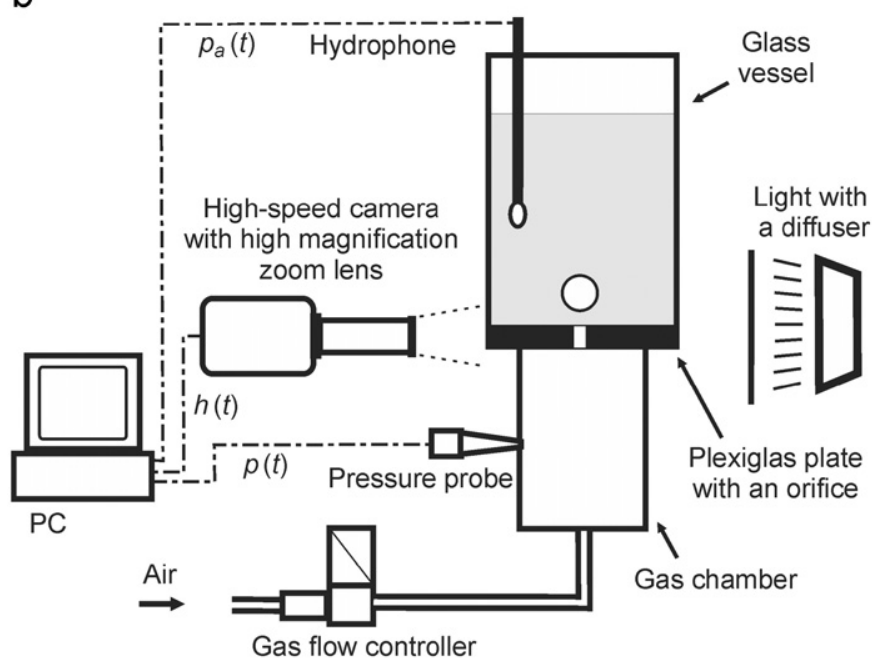

Fig. 1. Schematic diagram of the experimental apparatus: (a) the measuring cell and (b) the complete setup. forming a bubble (volume $V_{b}$ ) with the total bubbling period $T$ and the corresponding bubbling frequency $1 / T$.

The following physical signals were taken in the measurements. The gas pressure $p(t)$ was recorded in the chamber (pressure transducer Kistler, Kristal 4394 A0.2). The acoustic pressure $p_{a}(t)$ was monitored in the liquid (hydrophone Brüel \& Kjær, type 8103 with amplifier Nexus 2692 A0S4). The meniscus position $h(t)$ inside the orifice was video-recorded (high-speed camera Photron Fastcam SA1.1, zoom lens Navitar, up to 5400 fps at 1 Mpixel resolution). Also, the video of the growing and detaching bubble was taken. All the signals were synchronized with the high-speed camera, using the LabVIEW environment.

The uncertainty of the meniscus position $h(t)$ was estimated from the precision of the $h(t)$ position evaluation and the camera resolution. The camera resolution was generally $\sim 4.7 \mu \mathrm{m}$ per pixel. The meniscus was identified with the precision 2 pixels in the focal plane, which was set in the axis of the orifice; hence the uncertainty of $h(t)$ was roughly $10 \mu \mathrm{m}$. Usually, the formation of 10 bubbles was recorded by high-speed camera at typical rate $1000 \mathrm{fps}$, so the time variation was $\pm 1 \mathrm{~ms}$. The mean values of the quantities obtained from the records of the meniscus motion were averaged over three realisations. The pressure record $p_{a}(t)$ and $p(t)$ was at least $25 \mathrm{~s}$ long, which corresponds to $\sim 20-440$ realisations.

From these signals, several useful state variables were extracted. The total bubbling period $T$ and frequency $f$ can be obtained from all the signals, $p, p_{a}$, and $h$. The period $T_{A}$ for which the orifice is open and the gas flows through it into the bubble (active orifice time), as well as the period $T_{B}$ when the orifice is closed and the plenum pressurises (waiting period), follow from both $p$ and $h$, respectively. The details of the meniscus behaviour can be obtained from the signal $h$ : number $N$ of meniscus oscillations, their amplitudes $A_{n}$ and periods $T_{n}$, the total time of oscillation $T_{o}$, the rest time $T_{r}$ between the end of the oscillations and new orifice opening, and the total time $T_{B}$ when the orifice is closed. Note that $T=T_{A}+T_{B}$ and $T_{B}=T_{o}+T_{r}$. Fig. 2 shows the diagram with the periods and also pictures demonstrating the visualisation.

In this study, the effect of the following five operational parameters were considered: orifice size $d$, plate thickness $L$, gas chamber volume $V$, liquid height $H$, and liquid viscosity $\mu$. In our previous study (Ruzicka et al., 2009a, b; Ruzicka, 2009), they had the following fixed values: $d=1.6 \mathrm{~mm}, L=3 \mathrm{~mm}, V=307 \mathrm{ml}$,

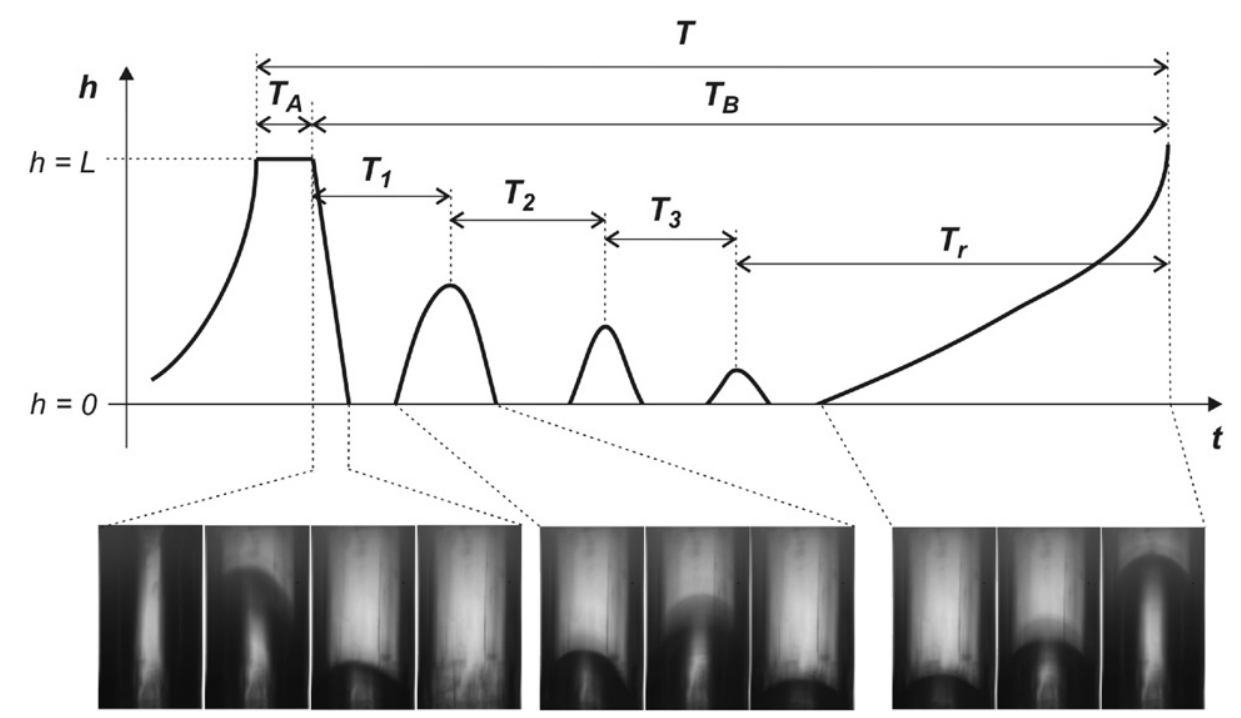

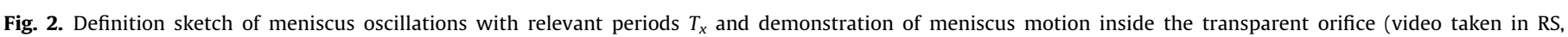
$Q=3 \mathrm{ml} / \mathrm{s}$ ). 
$H=6 \mathrm{~cm}$, and $\mu=1 \mathrm{mPa}$, which are taken here as the Reference State (RS). In the present parametric study, they have the following values (with the same units): $d=0.5,1,1.6^{*}, 2,4.8 ; L=3^{*}, 6$, $12 ; V=153,307^{*}, 614 ; H=6^{*}, 60$, and $\mu=1^{*}, 2.6,7,13,21,38,61$, 120 , and 211 , where the star * indicates the reference value of RS. To get the desired effect in the pure form, we tried to change only one parameter at the same time, while the others were kept in RS. Besides these five parameters, the main control quantity was the gas input $Q$ taking about 50 different values within the range $Q=0.42-8.33 \mathrm{ml} / \mathrm{s}$, to secure the 'bubbling mode' of bubble formation (e.g. Ruzicka et al., 1997).

\section{Results and discussion}

\subsection{Influence of orifice diameter d}

The orifice width $d$ is an important parameter for the bubble formation and meniscus dynamics, because it enters the criteria that determine the mode of bubbling and the occurrence of weeping. Subsequently, it affects the resulting bubble size and the quality of the gas dispersion, whence the structure of the gasliquid mixture. Five values of the orifice width were tested, $d=0.5,1,1.6^{*}, 2$, and $4.8 \mathrm{~mm}$. The reference ${ }^{*}$ value $d=1.6 \mathrm{~mm}$ diameter was chosen in accordance with the size of holes drilled in some perforated plates used in our bigger-scale gas-liquid equipment (bubble columns and airlifts) and with our previous measurements. The other values of $d$ were chosen to have both smaller and larger sizes, to see the trends roughly within one order of magnitude. The results can be summarised as follows.

With a medium orifice ( $d=1.6 \mathrm{~mm}$ ), we had a well-developed dome-shaped meniscus. After the bubble detachment, the meniscus dropped completely down, from the upper $\operatorname{rim}(h=L=3 \mathrm{~mm})$ to the lower rim (lower rim, $h=0$ ) and performed several damped oscillations around this equilibrium position. No apparent weeping was observed. With a smaller orifice $(d=1 \mathrm{~mm})$ and low $Q$, the meniscus dropped down only partly, by $\approx 1 \mathrm{~mm}$ and performed few fast oscillations around this elevated position $(h \approx 2 \mathrm{~mm})$. The amplitude was low, i.e. the meniscus was flat. Then the meniscus acquired the domical shape and started to move upwards to open the orifice. With increasing $Q$, the equilibrium moved upwards and at about $Q \approx 4 \mathrm{ml} / \mathrm{s}$ it reached the upper rim, where it stayed also for higher gas inputs. With an even smaller orifice $(d=0.5 \mathrm{~mm})$, we encountered optical problems at video-recording. The cylindrical surface had such a large curvature that the strong reflection made it almost non-transparent. We tried several types of lights of high intensity (metal-halide lamp with optical fibres, strong halogen lamp, super LED diodes, etc.), but we failed at illuminating the meniscus sufficiently for recording its motion properly. With a larger orifice $(d=2 \mathrm{~mm})$, the meniscus could stand the static load at no gas throughput, when the pressure equilibrium was reached (gas below/liquid above). Under dynamical conditions, with the gas input, the liquid leaked slowly through the orifice. With an even larger orifice $(d=4.8 \mathrm{~mm})$, pronounced leakage was encountered.

These results can be discussed in terms of two dimensionless numbers, namely the Bond (Eotvos) number and the Weber number. The Bond number is $B o=(\Delta) \rho g X^{2} / \sigma$, where $X$ is the relevant length-scale of the system. $B o$ is the ratio of the gravity $(\rho)$ or buoyancy in two phase systems $(\Delta \rho)$ forces to the capillary forces, and is a relevant parameter when these two interplay (usually static or quasi-static problems). The reasonings involving $B o$ apply only for low gas flow through the orifice (e.g. interval $T_{B}$, interval $T_{A}$ at low $Q$ ), to avoid strong dynamical effects. The gravity prevails at $B o>1$ while the capillarity dominates at $B o<1$. Putting $B o=1$, we can estimate the particular length-scale at which the two effects are comparable. This scale is called the capillary length ${ }^{1}$ and for our case, $\lambda=(\sigma / \rho g)^{0.5} \approx 2.7 \mathrm{~mm}$. With the meniscus inside the orifice, the relevant scale is the orifice diameter. Therefore, our system is gravity-controlled at $d>\lambda$ and capillary-controlled at $d<\lambda$. The former (high $d$ ) means that the contact line and the Laplace pressure cannot support the liquid mass above the meniscus, whence the gravity weeping. The latter (low $d$ ) means that the meniscus can be formed but does not oscillate, and its behaviour is strongly affected by the material properties of the plate. With highly hydrophilic orifices, we can have the capillary weeping. With hydrophobic plates, the liquid may not even enter the orifice. When $d \sim \lambda$ are comparable by the order of magnitude, both counter-acting gravity and capillary forces are presented and yield oscillations. This picture parametrised by $\mathrm{Bo}$ is consistent with the results obtained in our measurements.

Another criterion is the Weber number, $W e=(\Delta) \rho X U^{2} / \sigma$, where $U$ is the relevant velocity-scale of the system. We is the ratio of (inertia/capillarity) forces, when these two interplay (usually dynamic problems). The inertia prevails at $W e>1$ while the capillarity dominates at $W e<1$. The former means that the meniscus is destroyed by the fluid (gas or liquid) stream. The latter means that the meniscus can exist. With the meniscus, the relevant scales are the orifice diameter ( $X$-scale) and the speed ( $U$-scale) of the meniscus (interval $T_{B}$ ) or speed of inflowing gas (interval $T_{A}$ ) or speed of inflowing liquid (early stage of $T_{B}$ ). For our case, $W e=22 U^{2}$.

First, the meniscus speed during its motion in the waiting period (interval $T_{B}$ ) does not typically exceed $u \sim 10^{-1} \mathrm{~m} / \mathrm{s}$. Whence We $\sim 10^{-1}$ showing that the system is capillary-controlled, which means that a stable meniscus inside exists and can oscillate. In very narrow orifices, the oscillations are suppressed, since the meniscus 'scrapes' against the wall. This was also observed in the measurements.

Second, the gas speed in the orifice during the bubble inflation (interval $T_{A}$ ) can be estimated from the bubble volume, $V_{b}=Q T=Q_{A} T_{A}$, where the actual orifice gas flow is $Q_{A}=u S$, whence $u=\left(T / T_{A}\right)(Q / S)$. In our measurements (in RS), in the direction of increasing $Q$, the value $T$ drops from 1.14 to $0.063 \mathrm{~s}$ (Fig. 4a), value of $T_{A}$ keeps at $\approx 0.03 \mathrm{~s}$ (Fig. $4 \mathrm{~b}$ ), parameter $Q$ changes within the range $0.42-8.33 \times 10^{-6} \mathrm{~m}^{3} / \mathrm{s}$, and the orifice area is $S=2 \times 10^{-6} \mathrm{~m}^{2}$. Resulting estimate of $u$ varies slowly from 8 (low $Q$ ) to 8.6 (high $Q$ ) $\mathrm{m} / \mathrm{s}$. Thus, we have $u \sim 10^{1} \mathrm{~m} / \mathrm{s}$, which is by two orders larger than the meniscus speed. Consequently, $W e \sim 10^{3}$, a value high enough to affect the meniscus, deform and possibly destroy. Here, at the upper rim, the gas penetrates the liquid to create a bubble or a jet: 'inverse weeping'. At lower We, the interface only deforms (expands) at the simultaneous formation of subsequent individual bubbles (bubbling mode), with meniscus oscillations after the detachment. At higher We, the interface is disintegrated by the continuous gas jet (jetting mode of bubble production) and no meniscus closes the orifice. This agrees with the results of the measurements. Note that in this study, we operate in the bubbling mode and the jetting mode was studied elsewhere (e.g. Ruzicka et al., 1997)

Third, after completing the necking process at the bubble detachment, the liquid stream flashes back inside the orifice, and the liquid piston falls down. During this fast drop, the meniscus speed reaches the peak values, up to about $1 \mathrm{~m} / \mathrm{s}$. Then, $W e \sim 10^{1}$, and the capillary forces at the lower rim are not strong enough to halt the liquid, whence the inertia weeping. This was in agreement with the measurements.

\footnotetext{
${ }^{1}$ Note a misprint in Ruzicka et al. (2009b, Section 2.2.2, p. 1360), where it is wrongly given as: $\lambda=(\rho g / \sigma)^{0.5}$.
} 
We can summarise the results and the discussion as follows. At low gas input (low We), the Bond number is relevant. With wide holes, (high $B o$ ), we have the gravity-driven weeping. With medium holes (medium Bo), we have the stable oscillating meniscus. With narrow holes (low Bo), the meniscus motion is strongly influenced by the material properties of the orifice and the dynamics of the contact line, since the ratio (surface)/ (volume) is typically very large for such a orifice. At high gas input (high We), not the Bond number but the Weber number is relevant. At relatively lower gas input (lower We), we have the bubbling regime where the meniscus closes the orifice, moves inside it, and possibly oscillates. At relatively higher gas input (higher $W e$ ), we have the jetting regime where the orifice is open constantly, and the meniscus does not exist. Also, when the inertia of the liquid piston at the lower rim is larger than the capillary forces, we have the inertia weeping. The result is summarised in Fig. 3a. Note also that the value $2.7 \mathrm{~mm}$ taken for the capillary length is only an order of magnitude estimate of
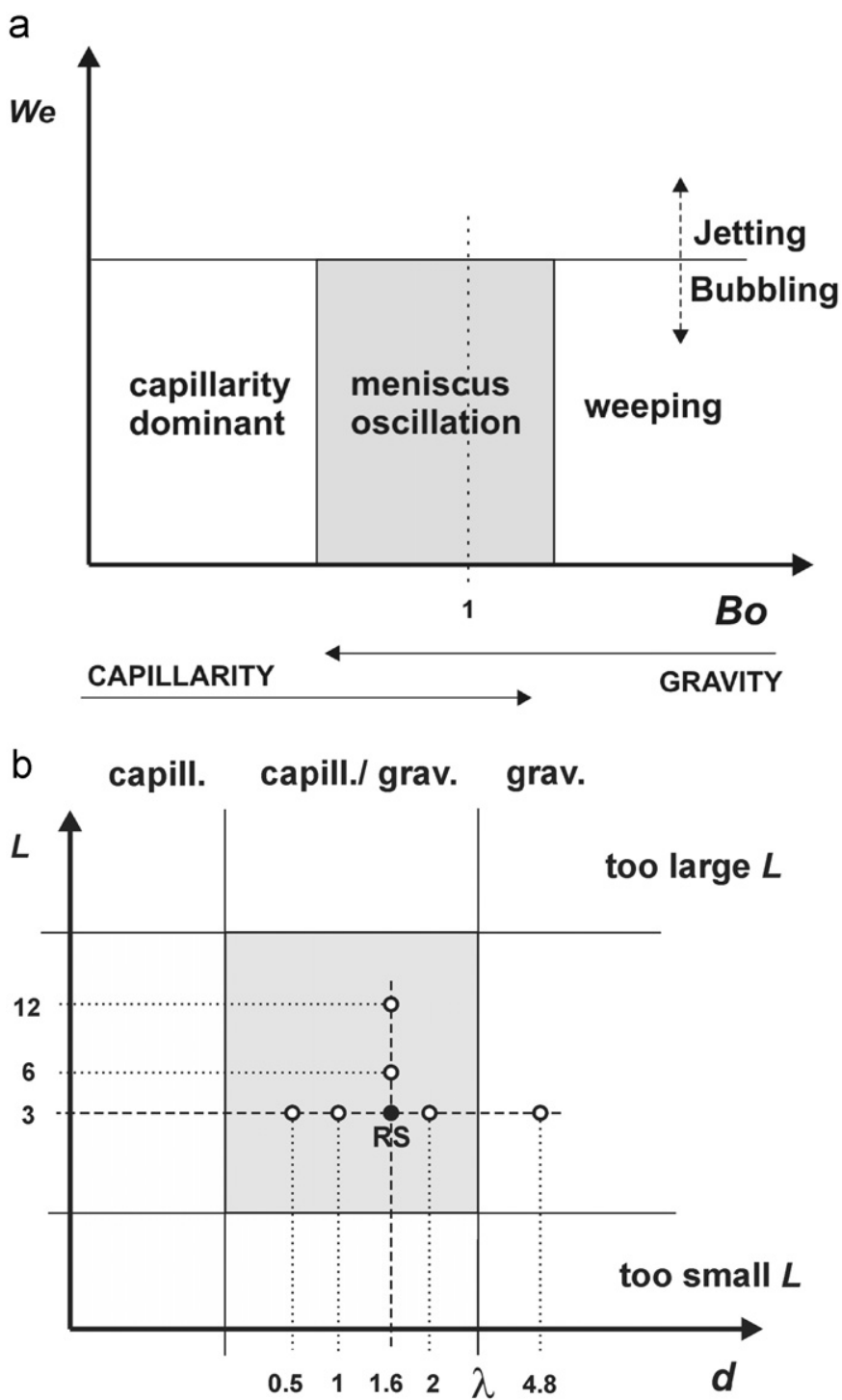

Fig. 3. (a) Effect of the orifice diameter $d$ on the bubbling process in terms of Bond and Weber numbers and (b) additional effect of the orifice length $L$ in the parametric plane $(d \times L)$. More complete would be a diagram in the parametric space $B o \times W e \times L$, with $L$ properly scaled for a particular process considered. Consequently, (a) and (b) are only two projections, where in the latter the limits on $d$ can be assessed as in Section 3.1 and those on $L$ are sketched only qualitatively. the characteristic length-scale over which the capillarity can compete with gravity. Similarly, such estimates are also the dimensionless numbers, $B o \sim X^{2}$ and $W e \sim X U^{2}$ (for a more detailed discussion see e.g. Ruzicka, 2008). Consequently, one cannot expect that exactly $B o=1$ is the strict border line between two regimes (recall e.g. laminar/turbulent flow transition occurring at $R e$ vastly unequal to unity). More important is the proper ordering of the subsequent regimes while changing the control parameter.

It is known that the orifice dimension is of high importance and this effect is often considered in the literature devoted to the bubble production, as correctly mentioned also in the review articles (see e.g. Kumar and Kuloor, 1970; Tsuge, 1986; Kulkarni and Joshi, 2005). The important phenomenon of weeping is not a subject of this study and was mentioned here only marginally to set some limits on the regions of the meniscus existence. There are however several detailed studies dealing with the weeping in the literature (e.g. McCann and Prince, 1969; Thorat et al., 2001).

\subsection{Influence of plate thickness $L$}

The plate thickness (orifice length, height) $L$ is an important parameter for the bubble formation and the meniscus dynamics. Together with the width $d$, it determines the orifice geometry (dimension and aspect ratio), whence the orifice working mode. The orifice width $d$ is directly related to the capillary phenomena, since the meniscus spreads over the orifice cross-section and its periphery and the contact line touches the wall at the circle. The orifice length $L$ is the meniscus travel at its piston-like motion. Three values of $L$ were tested: $L=3^{*}, 6$, and $12 \mathrm{~mm}$. The reference value $3 \mathrm{~mm}$ diameter was chosen in accordance with perforated plates used in our other experiments. The other values are larger, since reducing the plate thickness far bellow few $\mathrm{mm}$ is rather impractical. The results can be summarised as follows.

We observed a general trend in the equilibrium position of the meniscus, after the bubble detachment. While with the thinnest plate $(L=3 \mathrm{~mm})$, after the oscillations died out, the meniscus settled at the lower orifice rim $(h=0)$; with the medium plate ( $L=6 \mathrm{~mm}$ ) it settled roughly in the middle of the orifice height $(h \approx L / 2=3 \mathrm{~mm})$. With the thickest plate $(L=12 \mathrm{~mm})$, the meniscus dropped about $3 \mathrm{~mm}$ inside the orifice and most of the time stayed in its upper region. With increasing gas input $Q$ these trends were even more pronounced. Also, the vigour of the oscillations was weakened by increasing the plate thickness.

To discuss, following the piston-in-cylinder mechanical analogy established previously, the cylinder length increases while its width is fixed. This increases the resistance force and suppresses the oscillations, as observed. The fact that the meniscus equilibrium position was not much affected with $L$ may be explained by the pressure equilibrium between the plenum and the hydrostatic head, where the gas phase can be considered as incompressible, under given circumstances.

The joint effect of both $d$ and $L$ is sketched in Fig. 3b. The meniscus can exist within the range where both $d$ and $L$ are moderate. Orifice diameter $d$ should not be much lower and not much higher than the capillary length. Plate thickness $L$ should not be too low (no piston in a zero-thickness plate) and not too high (capillary regime at low $d$ and gravity regime at high $d$ ). The specific shape of the region boundaries will depend on the other quantities that are involved in the process and are under given conditions relevant. The increasing orifice length effect on dampening the piston oscillations due to the resistance force can be modelled with help of the Reynolds number to reflect the macroscopic aspects and with the dynamics of the triple line to reflect the microscopic aspects (e.g. de Gennes et al., 2004). 
When the ratio $(L / d)$ is very large, the plenum buffering effect is diminished and the orifice behaviour tends to the limiting case of a thin long needle (capillary). This case is not considered here and is treated elsewhere (e.g. Oguz and Prosperetti, 1993). A brief note about the influence of $L$ is e.g. in Kumar and Kuloor (1970), but probably there is a need for a more systematic study.

\subsection{Influence of gas chamber volume $V$}

The gas plenum volume is an important parameter for the bubble formation and the meniscus dynamics. It is the capacity term inserted between the gas source (pressure cylinder) and the gas sink (bubble). Its size affects the gas transport to the orifice and through it, and thus influences the bubble growth process. Three values of $V$ were tested, $V=153,307^{*}$, and $614 \mathrm{ml}$. The reference value ( $307 \mathrm{ml}$ ) was selected in accord with our previous measurements. The lower $(153 \mathrm{ml})$ and higher $(614 \mathrm{ml})$ values differ for RS roughly by factor two. These values are located in the 'medium' size region, with the volume ratio (chamber/bubble) $\sim\left(10^{2} \mathrm{ml} / 10^{0} \mathrm{ml}\right)$. A common criterion for assessing the chamber volume is based on the capacitance number, defined e.g. as (Park et al., 1977; Tsuge, 1986) $N_{C} \equiv 4 g \Delta \rho V / \pi \kappa p d^{2}$. It compares the actual chamber size $V$ with a certain 'critical' size derived from simplified kinematic and thermodynamic considerations ${ }^{2}$. There are several interpretations of this ratio: one can see the buoyancy force on the gas chamber $(g \Delta \rho V)$, the gas pressure force $((1 / 4)$ $\left.\pi d^{2} p\right)$ on the orifice cross-section, etc. For our three choices of $V$, we have $N_{C}$ roughly about ten (namely $N_{C}=5.2,10.5$ and 20.9). Since many effects interplay, the medium range is the most complex, whence most challenging. Also, in applications, we usually have a medium size plenum (far from zero and infinity).

The results are shown in Figs. 4 and 5. The effect of plenum size $V$ on the mean value of total bubbling period $T$ is shown in Fig. 4a, where it is plotted versus the gas input $Q$, with $V$ as the parameter. The period drops with $Q$ and grows with $V$. In all three cases, the graphs well obey a power-law fit, $T=a Q^{b}$, where the exponent $\mathrm{b}$ is close to unity and does not vary much $V$, see $b=-0.92,-0.98$, and -1.02 in Table 1 . Thus the roughly hyperbolic character of the $T(Q)$ dependence does not change with the chamber volume. The increase of $T$ with $V$ is reflected by increasing the proportionality coefficient: $a=376,516$, and 606, see Table 1 . The increment of $T$ with $V$ has a decreasing trend (Table 1 ), from which it can be predicted that the period will reach a saturation value at a large enough plenum (constant pressure condition). The effect of plenum size $V$ on the active orifice time $T_{A}$ is shown in Fig. $4 \mathrm{~b}$. The period $T_{A}$ shows no apparent trend with gas input $Q$ and increases with $V$, while the increment has a diminishing trend (Table 1). The saturation of $T_{A}$ value at a large enough $V$ can thus be expected.

These results can be discussed together with the bubble size. The mean bubble volume $V_{b}$ can be calculated as $V_{b}=Q T$, using the total bubbling time $T$. Using our data, this formula gives $V_{b}=a Q^{1+b} \approx a$, so that the bubble size is not much sensitive to $Q$ in force of $b \approx-1$.

At low $Q$, the observations can be explained by the time-scale decoupling between the two processes: plenum filling (time $T_{B}$ ) and plenum emptying (time $T_{A}$ ). When $T_{A}<T_{B}$, the bubble forms almost instantly with respect to the long time needed to refill the plenum. The amplitude of the pressure signals (discussed in previous studies) is the plenum pressure drop $\Delta p$, which is the driving force of the gas flux through the orifice into the bubble, is

${ }^{2}$ Note that this criterion serves as only a rough assessment of certain aspects of the gas chamber role in the bubbling process and that similar 'capacitance' considerations can be employed also for capillaries (e.g. Vejrazka et al., 2008).

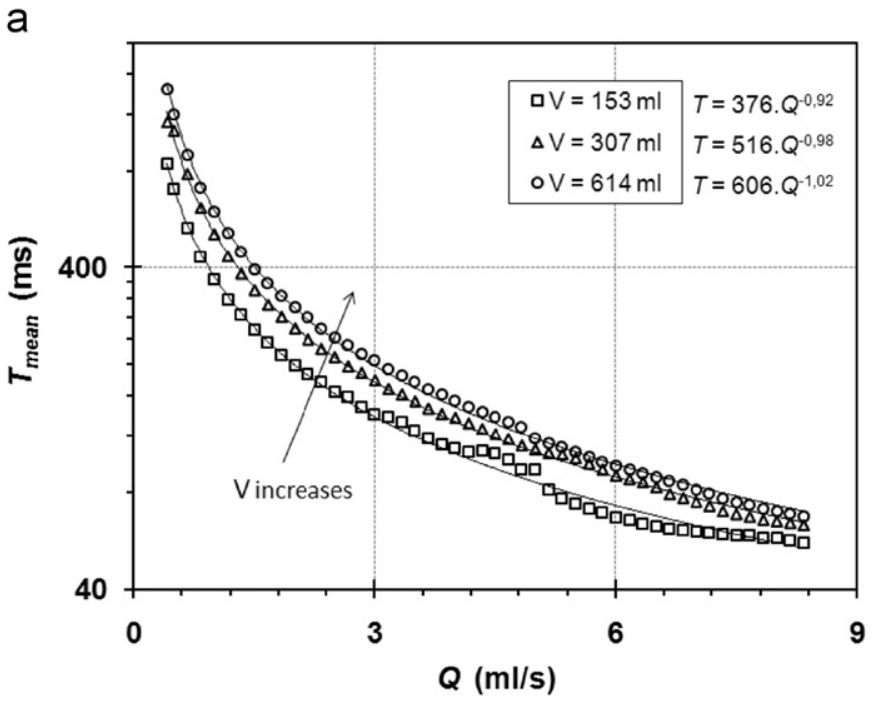

b

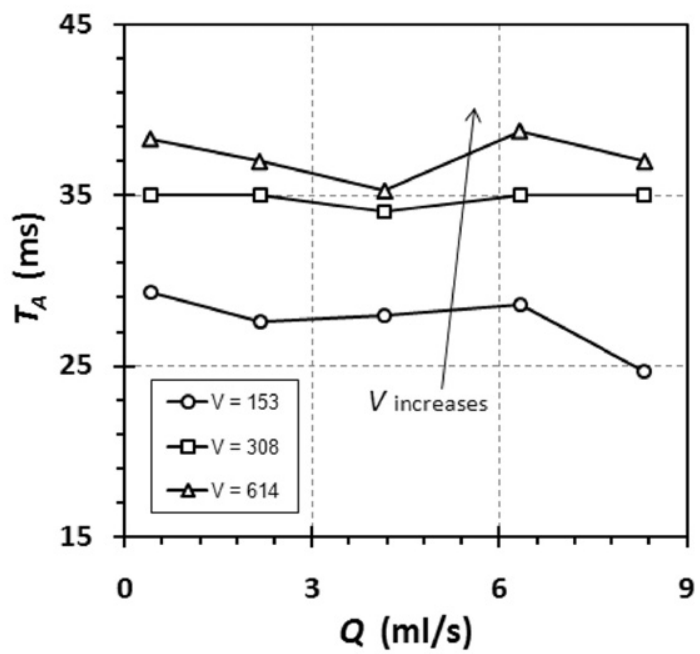

Fig. 4. Effect of gas plenum size $V$ : (a) mean values of total bubbling period $T$ against gas input $Q$ (mean of 25-400 samples for each $Q$ ) and their power-law trends $T=a Q^{b}$ and (b) active orifice time $T_{A}$ (mean over 3 samples) against gas input $Q$ for three values of $V=153,307$, and $614 \mathrm{ml}$.

nearly constant. At a fixed plenum size, the same amount of gas (corresponding to $\Delta p$ ) is transported by the same driving force from plenum to bubble. Consequently, it takes the same time $T_{A}=$ const. and gives the same bubble $V_{b}=$ const. At a larger plenum, at a comparable driving force $\Delta p$, larger amount of gas is transferred to the bubble, which takes a longer time $T_{A}$ and gives a bigger bubble $V_{b}$, as found in the measurements. The waiting time $T_{B}$, which here roughly is the total time $T$, can be estimated by the scale equation for plenum filling as $T \sim(\Delta P / P)(V /$ $Q) \sim Q^{-1}, V^{1}$, where the observed trend $T \sim Q^{-1}$ is correctly recovered by the data. The scale-estimated seemingly linear increase $T \sim V^{1}$ has to be corrected by considering the fact that the measured pressure difference $\Delta p$ decreases with the plenum size (see Table 1), to get the above expected saturation of $T$ with $V$.

The bubble size can also be calculated as $V_{b}=Q_{A} T_{A}$, using the orifice active time $T_{A}$. At a fixed plenum, bubble size is constant as well as the period $T_{A}$, whence the actual orifice flow $Q_{A}$ must be constant too. Note that $Q_{A}$ is difficult to measure directly, so any information extracted from the experiments is valuable. At a larger plenum, both $V_{b}$ and $T_{A}$ increase. If the increase of $T_{A}$ is the same as that of $V_{b}$, then $Q_{A}$ is constant again (Table 1 ). 
a

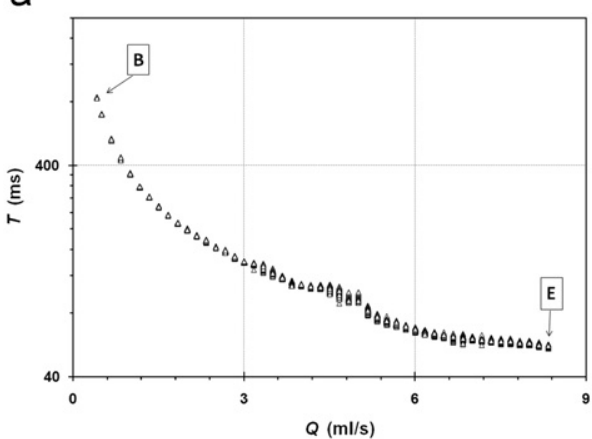

b

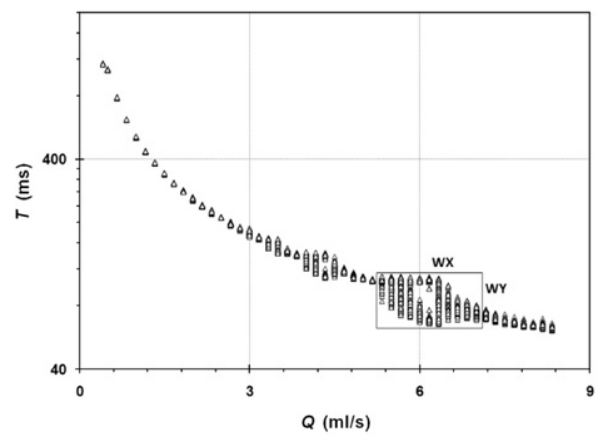

C

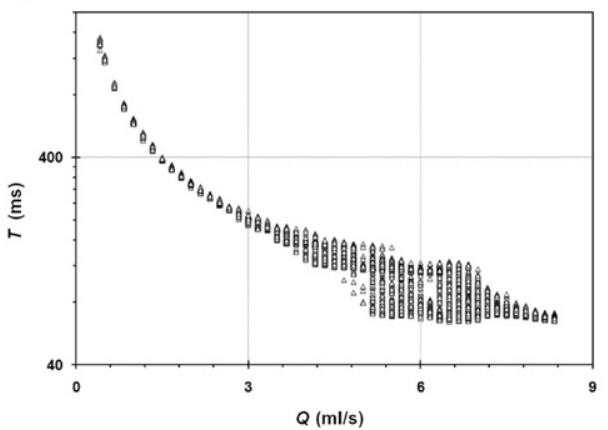

d

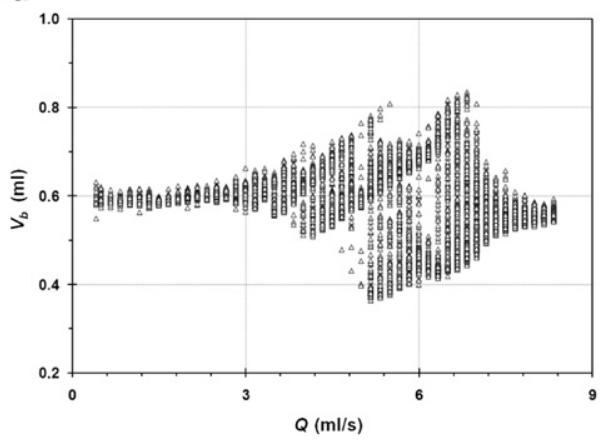

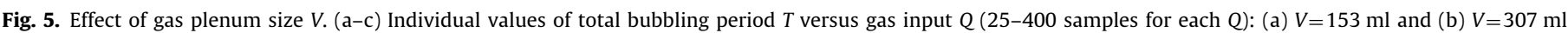

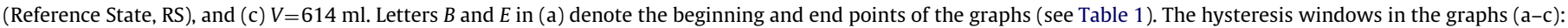

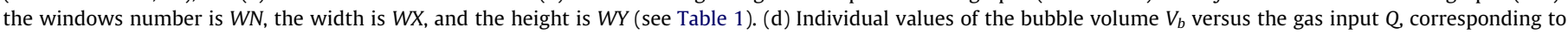
diagram (c).

\section{Table 1}

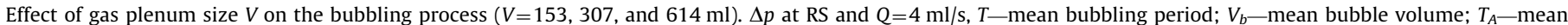

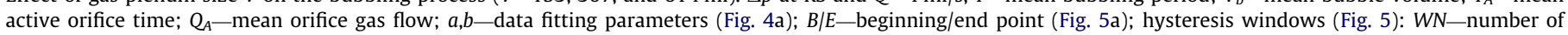
windows, $W X$ - window width, and $W Y$-window height.

\begin{tabular}{|c|c|c|c|c|c|c|c|c|c|c|c|c|}
\hline $\boldsymbol{V}(\mathrm{ml})$ & $\Delta \boldsymbol{p}(\mathrm{Pa})$ & $\boldsymbol{T}_{\text {mean }}(\mathrm{ms})$ & $\boldsymbol{V}_{\boldsymbol{b} \text { mean }}(\mathrm{ml})$ & $\boldsymbol{T}_{\boldsymbol{A} \text { mean }}(\mathrm{ms})$ & $\boldsymbol{Q}_{\boldsymbol{A} \text { mean }}(\mathrm{ml} / \mathrm{s})$ & $\boldsymbol{a}(\mathrm{mlms} / \mathrm{s})$ & $\boldsymbol{b}$ (dimensionless) & $\boldsymbol{B}(\mathrm{ms})$ & $\boldsymbol{E}(\mathrm{ms})$ & $\boldsymbol{W N}$ (dimensionless) & $\boldsymbol{W} \boldsymbol{X}(\mathrm{ml} / \mathrm{s})$ & $\boldsymbol{W} \boldsymbol{Y}(\mathrm{ms})$ \\
\hline 153 & 285 & 101 & 0.42 & 28 & 15.1 & 376 & -0.92 & 838 & 56 & 2 & 0.6 & 6 \\
\hline 307 & 220 & 127 & 0.53 & 35 & 15.2 & 516 & -0.98 & 1137 & 63 & 3 & 0.5 & 13 \\
\hline 614 & 130 & 141 & 0.59 & 37 & 15.9 & 606 & -1.02 & 1429 & 67 & 4 & 0.6 & 33 \\
\hline
\end{tabular}

At high $Q$ the time scale of the two processes are comparable $T_{A} \sim T_{B}$ and they can be strongly coupled. This occurs roughly for $Q$ higher than about $3 \mathrm{ml} / \mathrm{s}$. It is then difficult to treat the both processes separately and repeat the above reasonings. One important consequence of the plenum-bubble coupling is the hysteresis phenomenon, which was discovered and analysed in our previous study. The increasing plenum pressure interferes strongly with the meniscus oscillations and the interplay of the plenum filling rate and the meniscus oscillation phase determines the size of the resulting bubble. Consequently, even when all control parameters are kept fixed ( $Q$ too), the bubble size can vary due to the nonlinear dynamics of the meniscus-plenum coupling. The dispersion of values of individual bubble size $V_{b}$ is transferred also on the corresponding dispersion of individual bubbling periods $T$. The regions with multiple values in the $T(Q)$ graphs are called the hysteresis windows. Fig. 5 shows the effect of the plenum size on the hysteresis regions. Here, the individual periods are plotted, to see the apparent ambiguity. Usually, only the mean values of the bubbling periods are reported in the literature, since it is easier to obtain. However, the averaging erases the fine information about the hysteresis (cf. Figs. 4 and 5). To characterize the graphs in Fig. 5, we detect their points of beginning (denoted as $B$ ) and end $(E)$, quantify the windows by their number $(W N)$, their width $(W X)$, and their height $(W Y)$, see Table 1 , where the effect of $V$ is clearly seen. With increasing $V$, the both end points $B$ and $E$ raise, but the first one, $B$, much strongly. The number of windows and their size also grow with $V$ and their location seems to move to the right. A practical consequence of this dynamic subtlety is that with larger plenum we like get a higher polydispersity in bubble sizes, right at the gas distributor (see Fig. 5d). This can be detrimental to the uniformity of bubbly layers in our equipments.

It is recognised that the gas plenum effect generally is an important and complex factor, as reflected by the amount of literature devoted to this subject as correctly reflected also in review papers (see e.g. Kumar and Kuloor, 1970; Tsuge, 1986; Kulkarni and Joshi, 2005). Regarding the hysteresis phenomenon, in our studies we detected the ambiguity in the values of the bubbling periods whence the bubble sizes in systems with the finite gas plenum, and related it to the meniscus behaviour. The ambiguity was also observed in systems with effectively zero plenum (needles and capillaries) and was treated using the nonlinear dynamic ansatz in terms of bifurcation sequences (see e.g. Badam et al., 2007 and the references there in). 
a

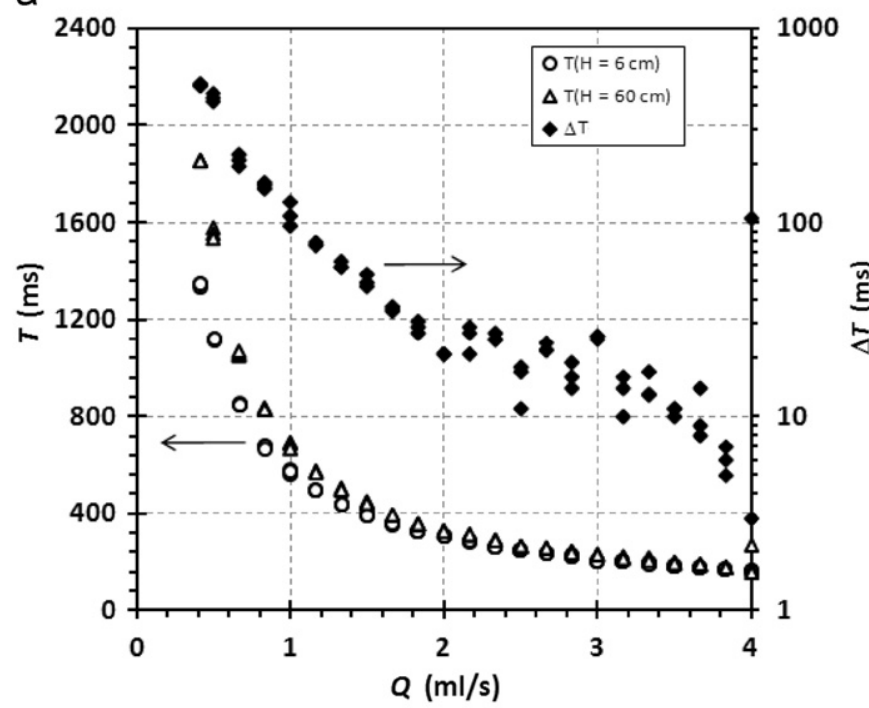

b

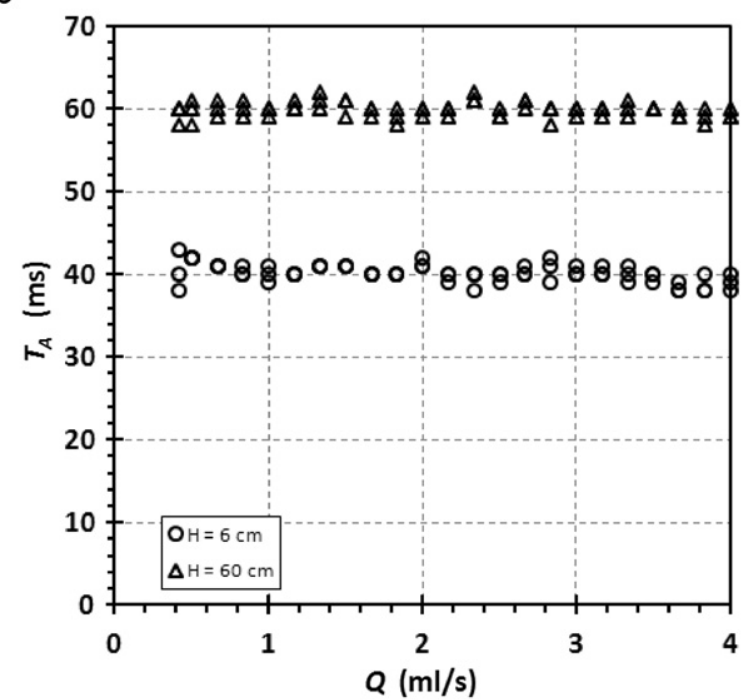

Fig. 6. Effect of liquid height $H$ : (a) total bubbling periods $T$ and their difference $\Delta T$ due to increasing $H$ versus gas input $Q$ and (b) active orifice time $T_{A}$ versus gas input $Q$. Liquid level $H=6$ and $60 \mathrm{~cm}$. Individual values of 3 samples.

\subsection{Influence of liquid height $H$}

The liquid height is an important parameter for the bubble formation and the meniscus dynamics. On the gas side, the hydrostatic head sets the pressure scale for the plenum. On the liquid side, it affects the size of the produced bubbles and the character of the bubble-induced liquid motions. In many applications, the ambient pressure is high, the column is very tall, and the liquid (metals) density is large; here the liquid level can be very important.

The reference height is $H=6 \mathrm{~cm}$, which seems to be sufficient for studying bubble formation. It is large enough and several-times the bubble size to have some liquid bulk. It is small enough to eliminate the large scale motions introduced by the subsequently rising bubbles, which are difficult to control and may affect the formation process on large time-scales, in a very unpredictable way. The test value was set ten-times higher, to $H=60 \mathrm{~cm}$, to assess the trend of influence. Although the factor of the parameter change here is $10 \times$, the change in the total pressure level is almost negligible. The ambient pressure is about $10^{5} \mathrm{~Pa}$, and the hydrostatic head in our column was roughly 600 and 6000 Pa (i.e. $0.6 \%$ and 6\%). Moreover, at any head, the plenum pressure must meet the hydrostatic pressure to ensure the equilibrium. In some aspects, the ambient gas pressure and the hydrostatic pressure are interchangeable, equivalent in their effect (e.g. thermodynamic and kinematic considerations). In another aspects they are not (dynamic considerations, momentum transmission due to expand bubble, liquid motions, hydroacoustics, etc.).

In our measurements, the bubbling at the elevated liquid level was accompanied with a small slow weeping, whose beginning was hard to observe due to its aperiodicity. The liquid volume wept during the relatively short period of measurement was negligible to the total $H$. The total bubbling period $T$ increased with the increase in the liquid height $H$, as seen from Fig. $6 a$. The difference $\Delta T$ is apparent at low gas input and falls quickly with $Q$. On the other hand, the active orifice period $T_{A}$, decisive for bubble size, is nearly constant within the whole range of $Q$ and is larger at the elevated height $H$, see Fig. $6 \mathrm{~b}$. The oscillation dynamics of the meniscus is expressed by the first three periods $T_{1,2,3}$ in Fig. 7 . At a larger hydrostatic head, the periods basically follows that of RS in their trends but are systematically slightly lower. Also, the oscillations extend to a shorter interval of $Q$.

To discuss, the oscillations at the elevated pressure level experience a stronger whence faster elastic response from the gas phase, and the time-scales tend to decrease. On the liquid side, the meniscus motion is projected also into the liquid bulk in the column above the plate. The larger the amount of the liquid affected the faster decay of the oscillatory motions. Also, while the wept volume was negligible with respect to the total liquid volume, its downward flux through the orifice could delay the gas up flow and suppress the meniscus motions.

It is likely that the effect of the orifice submergence has not been paid enough attention, as can be presumed from the published results, where the data at variance are not exceptional or even in a contradiction (see e.g. Kumar and Kuloor, 1970; Kulkarni and Joshi, 2005).

\subsection{Influence of liquid viscosity $\mu$}

The liquid viscosity is an important parameter for the bubble formation and the meniscus dynamics, since it affects many of the processes involved. It is the most often varying liquid property in practical applications, with a strong impact on the mode of the gas dispersion. The reference value $\mu=1 \mathrm{mPa} s$ is rather obvious, since it is water. Rarely we encounter much lower values for liquids in our engineering practise. On the contrary, very often we meet higher values. This was the motivation for choosing the set of the tested values, from $1 \times$ to roughly $200 \times$ the water viscosity. The viscosity effect was measured for two values of gas input, namely $Q_{1}=0.83 \mathrm{ml} / \mathrm{s}$ (low input, $10 \%$ of the largest) and $Q_{2}=5 \mathrm{ml} / \mathrm{s}$ (higher input, $50 \%$ of the largest).

The viscosity effect on the total bubbling period $T$ is shown in Fig. 8a. At higher $Q$ the effect is very weak, especially when considering the logarithmic scale of the ordinate. At lower $Q$, the period $T$ is larger (consistent with Fig. 4a) and more sensitive to the viscosity variation. The general trend is the overall increase in $T$, from about 600 to over $900 \mathrm{~ms}$.

In particular, the little hump at about $\mu \approx 3 \mathrm{mPa}$ can be related to some other observations reported in the literature, where some effects can come into force at studying the uniformity of bubbly layers and their stability about this specific value (e.g. see Fig. 4 in Ruzicka et al. (2003) and Fig. 4 in Olivieri et al. (2011)).

The viscosity effect on the orifice opening time $T_{A}$ is shown in Fig. 8b, which differ from $T$ in several aspects. First, $T_{A}$ is much lower than $T$. Second, $T_{A}$ is not much sensitive to gas flow $Q$. Third, 
a

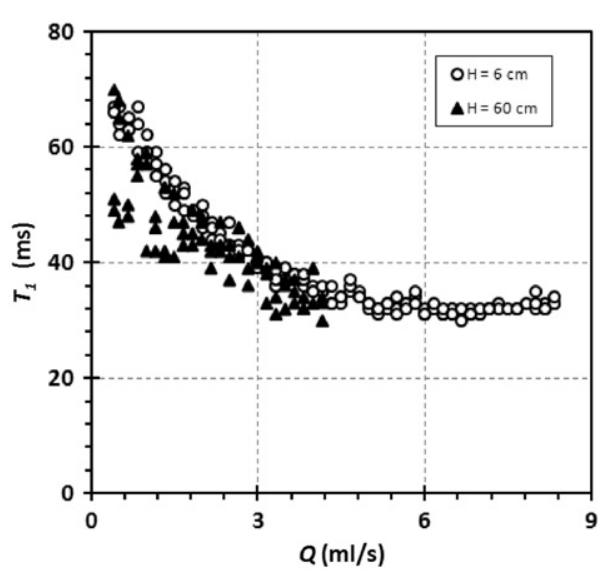

b

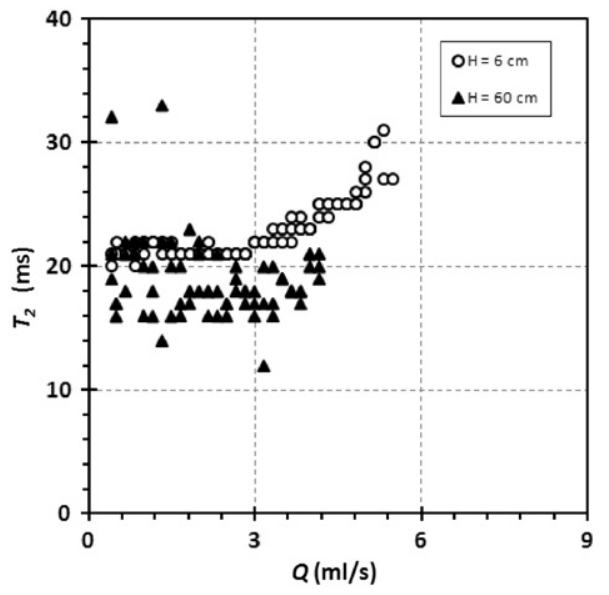

C

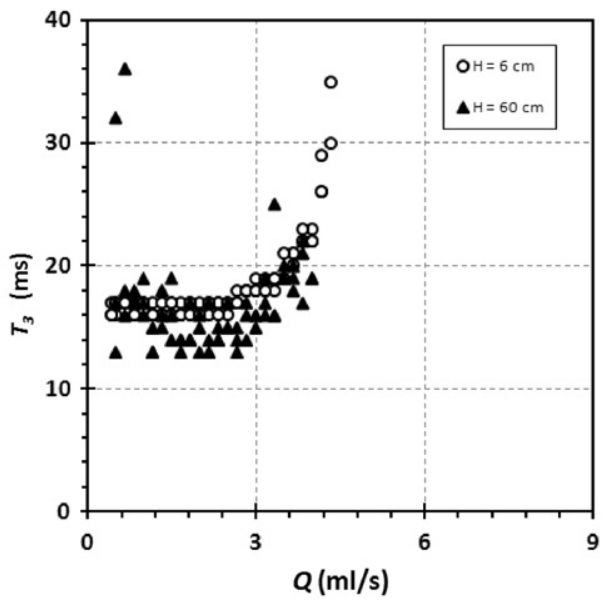

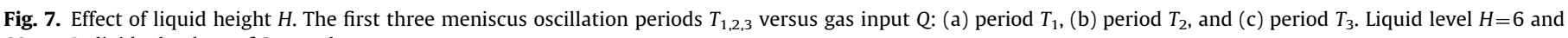
$60 \mathrm{~cm}$. Individual values of 3 samples.

$T_{A}$ has a more smooth trend of its progressive increase, from about 35 to $60 \mathrm{~ms}$. We see the marked difference between the behaviour of $T$ and $T_{A}$, the former being the usually reported quantity in the literature, but the latter being much more closely related to the bubble formation phenomena.

The viscosity effect on the individual periods of the meniscus oscillation is shown in Fig. 8c. A lot of information is contained in this small diagram. First, consider the global features. Typically, the ordering of the periods is the following: $T_{1}>T_{2}>T_{3}$. All oscillations disappear at about $10 \mathrm{mPa} s$ (no periods shown further). The number of oscillations (periods shown) decreases with $\mu$. Second, some more details can be retrieved too. At lower $Q$, the value of $T_{1}$ slightly increases with the viscosity and survives till $\mu \approx 3 \mathrm{mPa}$ s. $T_{2}$ is nearly constant and lasts for the same range. $T_{3}$ is the lowest and occurs only at the smallest viscosity $\mu=1$. At higher $Q$ the value of $T_{1}$ is almost constant, lower as compared with lower $Q$ and lasts till about $10 \mathrm{mPa} \mathrm{s} . T_{2}$ is comparable with that of lower $Q$ shows a slight raise, and decays at about $3 \mathrm{mPa}$. $T_{3}$ is comparable with that of lower $Q$ and occurs only at the smallest viscosity $\mu=1$.

To discuss, the inertia forces are stronger at higher $Q$ and the viscosity has lower possibility to affect the system (Fig. 8a). The dynamical effect of the viscosity is to slow down any motion, which results in a general trend to enlarge the characteristic timescales in the system (Fig. 8a and b). Dissipation effect is also important, which results in faster decay of the oscillatory motions (Fig. 8c).
It follows from the literature, that the effect of the liquid viscosity on the bubble generation process is not fully understood yet. It is symptomatic that the published results are not in full accord and may even be in a contradiction, as is discussed in some review contributions (see e.g. Kumar and Kuloor, 1970; Kulkarni and Joshi, 2005).

\section{Conclusion}

It was proved that the gross macroscopic description of the bubble formation process in terms of the mean overall quantities is insufficient for the deeper understanding of this important phenomenon. In particular it is insufficient to explain the way of the dynamical coupling between the gas (below the plate) and the liquid (above the plate) sides of the gas-liquid dispersing system. In our previous study, we developed a new concept of the gas-liquid coupling by resolving the behaviour of the meniscus inside the orifice during the bubbling process. In this study, we performed experiments focused on the effect of five important operational parameters on the meniscus dynamics: gas input, orifice size, plate thickness, gas plenum size, liquid height, and liquid viscosity. From the results obtained, the following conclusions can be made:

The effect of the orifice diameter $d$ can be to a large degree captured by the Bond number and the Weber number, which delimitate the prevailing meniscus mode in the parametric plane gravity versus inertia effects (Fig. 3a). 
a

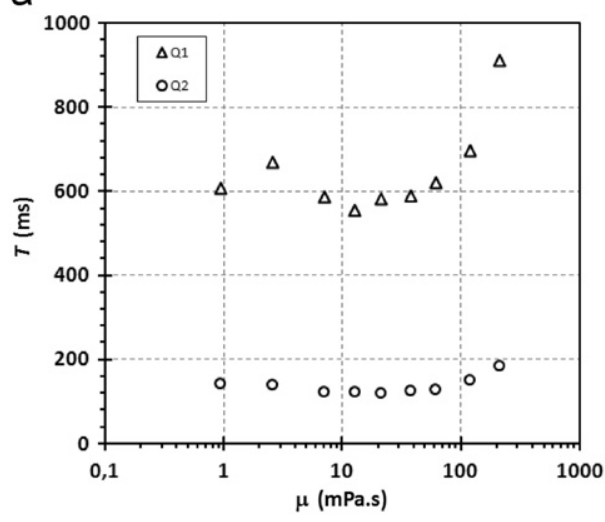

b

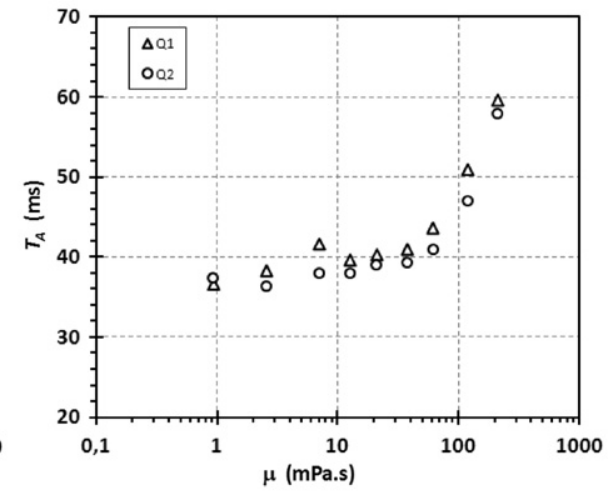

C

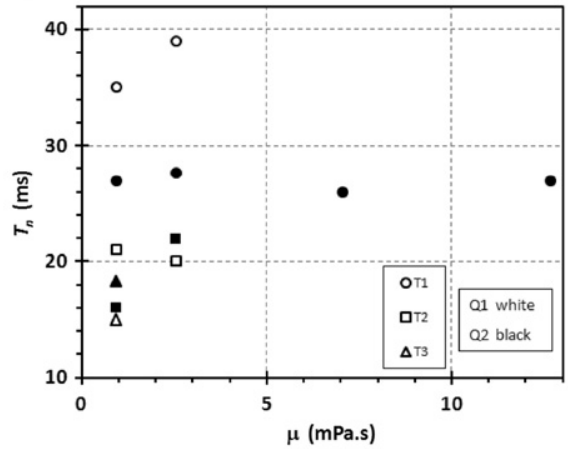

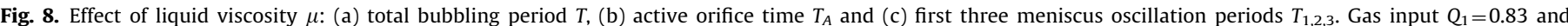
$Q_{2}=5 \mathrm{ml} / \mathrm{s}$. Mean values of 3 samples.

The effect of the plate thickness $L$ is not primarily involved in the capillary scaling but together with the orifice width it specifies the orifice geometry and determines the regions of the capillary/gravity modes (Fig. 3b).

The effect of the gas chamber size $V$ is complex and plays a crucial role at the meniscus motion. Reported are the increasing trends of the total bubbling period $T$ and the active orifice time $T_{A}$ when enlarging the plenum. Resolved are the mean values and the individual values of $T$, the latter showing a marked dispersion of values (denoted as "hysteresis"). This ambiguity is projected also into the bubble sizes and results in a strong polydispersity of the produced bubble ensemble (Figs. 4 and 5).

The effect of the liquid height $H$ is apparent in modifying the time-scale of the system, where the typical bubbling times $T$ and $T_{A}$ are enhanced; while the meniscus oscillations are fasten and limited in extent (Figs. 6 and 7).

The effect of the liquid viscosity $\mu$ is found to be reduced by higher gas input for $T$, but unaffected for $T_{A}$. The general trend is to slow down the motions, enlarge the time-scales, and smear out the oscillations.

\section{Nomenclature}

$a, b \quad$ parameters of fitting trend line

Bo Bond number, dimensionless

$d \quad$ orifice diameter, $\mathrm{m}$

gravity, $\mathrm{m} / \mathrm{s}^{2}$

$h \quad$ meniscus position inside orifice, $m$

$H \quad$ liquid height, $\mathrm{m}$

$L \quad$ plate thickness, $m$

$N_{C} \quad$ dimensionless chamber volume, dimensionless $p \quad$ chamber pressure, $\mathrm{Pa}$

$P \quad$ pressure scale, $\mathrm{Pa}$

Q gas flow into chamber from external source, $\mathrm{ml} / \mathrm{s}$, or $\mathrm{SI}$

$Q_{A} \quad$ gas flow into bubble during interval $T_{A}, \mathrm{ml} / \mathrm{s}$, or SI

$S \quad$ orifice cross-section, $\mathrm{m}^{2}$

$t \quad$ time, $\mathrm{s}$

$T \quad$ total bubbling period (see Fig. 2), $\mathrm{s}$

$T_{A, B} \quad$ active and passive orifice time (bubble growth and orifice waiting), s

$T_{n} \quad n$th period of meniscus oscillation, $\mathrm{s}$

$u \quad$ specific speed in orifice, $\mathrm{m} / \mathrm{s}$

$U \quad$ velocity-scale, $\mathrm{m} / \mathrm{s}$

$V \quad$ gas chamber volume, $\mathrm{ml}$ or $\mathrm{SI}$

$V_{b} \quad$ bubble volume, $\mathrm{ml}$ or $\mathrm{SI}$

We Weber number, dimensionless

X length-scale, $\mathrm{m}$

\section{Greek letters}

$\Delta \quad$ delta, difference

$\kappa \quad$ polytrophic coefficient, dimensionless

$\lambda \quad$ capillary length, $\mathrm{mm}$

$\mu \quad$ liquid dynamic viscosity, mPa s

$\rho \quad$ liquid density, $\mathrm{kg} / \mathrm{m}^{3}$

$\sigma \quad$ surface tension, $\mathrm{N} / \mathrm{m}$

\section{Abbreviations}

$B, E \quad$ beginning/end point (see Fig. 5a)

$W N, X, Y$ window number, width, height (see Fig. 5b)

RS reference state (also denoted by ${ }^{*}$ ) 


\section{Acknowledgements}

The financial support by the Grant Agency of the Czech Republic (GACR Grant no. 104/07/1110) and the Grant Agency of the Academy of Sciences of the Czech Republic (GAAV Grant no. KJB200720901) is gratefully acknowledged.

\section{References}

Badam, V.K., Buwa, V., Durst, F., 2007. Experimental investigations of regimes of bubble formation on submerged orifices under constant flow condition. Can. J. Chem. Eng. 85, 257-267.

Clift, R., Grace, J.R., Weber, M.E., 1978. Bubbles, Drops and Particles. Academic Press, New York.

Deckwer, W.D., 1992. Bubble Column Reactors. J. Wiley, Chichester.

de Gennes, P.G., Brochard-Wyatt, F., Quéré, D., 2004. Capillarity and Wetting Phenomena. Springer-Verlag, New York.

Kastanek, F., Zahradnik, J., Kratochvil, J., Cermak, J., 1993. Chemical Reactors for Gas-Liquid Systems. Ellis Horwood, Chichester.

Kulkarni, A.A., Joshi, J.B., 2005. Bubble formation and bubble rise velocity in gasliquid systems: a review. Ind. Eng. Chem. Res. 44, 5873-5931.

Kumar, R., Kuloor, N.R., 1970. The formation of bubbles and drops. Advances in Chemical Engineering, vol. 8. Academic Press, New York, pp. 255-368.

McCann, D.J., Prince, R.G.H., 1969. Bubble formation and weeping at a submerged orifice. Chem. Eng. Sci. 24, 801-814.

Mudde, R.F., 2005. Gravity-driven bubbly flows. Annu. Rev. Fluid Mech. 37, 393-423.
Oguz, H.N., Prosperetti, A., 1993. Dynamics of bubble growth and detachment from a needle. J. Fluid Mech. 257, 111-145.

Olivieri, G., Russo, M.E., Simeone, M., Marzocchella, A., Salatino, P., 2011. Effects of viscosity and relaxation time on the hydrodynamics of gas-liquid systems. Chem. Eng. Sci., 10.1016/j.ces.2011.01.027.

Park, Y., Tyler, A.L., de Nevers, N., 1977. The chamber orifice interaction in the formation of bubbles. Chem. Eng. Sci. 32, 907-916.

Ruzicka, M.C., 2008. On dimensionless numbers. Chem. Eng. Res. Des. 86 (8A), 835-868.

Ruzicka, M.C., 2009. Dripping faucet and bubbling faucet: an analogy. Chem. Eng. Res. Des. 87 (10), 1366-1370.

Ruzicka, M.C., Bunganic, R., Drahos, J., 2009a. Meniscus dynamics in bubble formation, Part I: experiment. Chem. Eng. Res. Des. 87, 1349-1356.

Ruzicka, M.C., Bunganic, R., Drahos, J., 2009b. Meniscus dynamics in bubble formation, Part II: model. Chem. Eng. Res. Des. 87, 1357-1365.

Ruzicka, M.C., Drahos, J., Zahradnik, J., Thomas, N.H., 1997. Intermittent transition from bubbling to jetting regime in gas-liquid two phase flows. Int. J. Multiphase Flow 23, 671-682.

Ruzicka, M.C., Drahos, J., Mena, P.C., Teixeira, J.A., 2003. Effect of viscosity on homogeneous-heterogeneous regime transition in bubble columns. Chem. Eng. J. 96, 19-26.

Thorat, B.N., Kulkarni, A.V., Joshi, J.B., 2001. Design of sieve plate spargers for bubble columns: role of weeping. Chem. Eng. Technol. 24, 815-828.

Tsuge, H., 1986. Hydrodynamics of bubble formation from submerged orifices. In: Cheremisinoff, N.P. (Ed.), Encyclopedia of Fluid Mechanics, vol. 3. Gulf Publishing Co., Houston, pp. 191-232.

Valentin, F.H.H., 1967. Absorption in Gas-Liquid Dispersions: Some Aspects of Bubble Technology. E\&FN Spon Ltd., London.

Vejrazka, J., Fujasova, M., Stanovsky, P., Ruzicka, M.C., Drahos, J., 2008. Bubbling controlled by needle movement. Fluid Dyn. Res. 40, 521-533. 